\title{
Cancer of the Corpus Uteri in White and Black Women in Michigan, 1985-1994
}

\section{An Analysis of Trends in Incidence and Mortality and Their Relation to Histologic Subtype and Stage}

\author{
Terri Madison, M.P... ${ }^{1}$ \\ David Schottenfeld, M.D., M.Sc. ${ }^{1}$ \\ Vicki Baker, M.D. ${ }^{2}$ \\ ${ }^{1}$ Department of Epidemiology, School of Public \\ Health, University of Michigan, Ann Arbor, Michi- \\ gan. \\ ${ }^{2}$ Division of Gynecologic Oncology, University of \\ Michigan Medical Center, Ann Arbor, Michigan.
}

The authors thank Peter DeGuire of the Michigan Department of Community Health for his assistance in providing registry data.

Address for reprints: David Schottenfeld, M.D., M.Sc., Department of Epidemiology, University of Michigan School of Public Health, 109 Observatory Street, Ann Arbor, Ml 48109.

Received February 25, 1998; accepted March 26, 1998.
BACKGROUND. Cancer of the corpus uteri occurs more commonly among white women in the U.S., yet survival is poorer for black women. This study examined whether this trend has changed and also examined the relation of age and histologic subtype to differences in stage.

METHODS. This retrospective cohort study assessed incidence trends, mortality trends, and the relation of age and histologic subtype to stage for 12,079 incident cases and 2325 deaths registered between 1985 and 1994 in Michigan. Rate ratios compared incidence and mortality. Odds ratios quantified the contribution of age and histologic subtype to differences in risk for advanced stage, using MantelHaenszel univariate techniques and multivariate logistic regression.

RESULTS. The overall incidence rate was 21.99 per 100,000 , and the overall mortality rate was 3.82 per 100,000 . Black women had a $40 \%$ lower risk (rate ratio [RR] $=0.60)$ of developing cancer of the corpus uteri but had a $54 \%$ greater risk $(\mathrm{RR}=$ 1.54) of dying from cancer of the corpus uteri. Black women were at greater risk of being diagnosed with either sarcoma or more aggressive adenocarcinoma. However, after adjustment for age and histologic subtype, black women still had an increased risk for advanced stage disease $(2.63,95 \%$ confidence interval $=2.19$ 3.16).

CONCLUSIONS. The disparity between white and black women persists in incidence and mortality trends for cancer of the corpus uteri. The greater frequency of more aggressive histologic subtypes experienced by black women accounts for only $10 \%$ of their excess risk for more advanced stage disease. Cancer 1998;83:1546-54.

(C) 1998 American Cancer Society.

KEYWORDS: endometrial neoplasms, uterine neoplasms, histology, incidence, mortality, black women, white women.

C ancer of the corpus uteri is the most common invasive gynecologic cancer occurring in women in the U.S., with an age-adjusted incidence rate of 21.3 per 100,000 and an age-adjusted mortality rate of 3.4 per $100,000 .{ }^{1}$ Of particular interest are the incidence and mortality trends for this cancer, namely, that incidence is higher among white women but mortality is higher among black women. Between 1988 and 1992, the age-adjusted incidence rate per 100,000 for white women was 22.3, as compared with 14.8 for black women. However, during the same time period, the age-adjusted mortality rate per 100,000 for black women was 6.0, compared with 3.2 for white women. In addition, between 1986 and 1991 the 5-year relative survival rate for white women was $85 \%$, but it was only $55.6 \%$ for black women. ${ }^{1}$ 
Several prognostic factors have been explored to assess whether differences in the distribution of these factors between black and white women can explain the survival discrepancy. Compared with white women, black women have poorer survival at age 65 years or older, ${ }^{1}$ have more advanced stage disease when diagnosed, ${ }^{1-7}$ have fewer well-differentiated tumors, ${ }^{2-8}$ and have a higher frequency of more aggressive histologic subtypes. ${ }^{3,6-8}$ However, in multivariate analysis controlling for these factors, the survival disparity persists. ${ }^{2-4,7}$ Moreover, the disparity in stage and poorer subsequent survival for black women does not appear to be due to decreased access to health care or delays in seeking treatment after initial symptom recognition. ${ }^{3,8,9}$

Two types of endometrial cancer have been proposed. Type I, or estrogen-dependent tumors, are more common and less aggressive. ${ }^{10-12}$ Type II, or estrogen-independent tumors, are relatively uncommon and appear to be more aggressive.$^{10-12}$ Using this division, type II endometrial cancers and uterine sarcomas together form a subgroup of more aggressive uterine cancers. Recently, it has been suggested that black women may experience a higher frequency of more aggressive uterine cancers, and this would explain the more advanced stage and poorer prognosis. ${ }^{3}$ However, much of the research regarding differences between black and white patients with this cancer has excluded sarcomas, and many of the study populations evaluated have been too small to assess carefully the racial differences among patients with uncommon type II histologic subtypes of disease. This study was undertaken to evaluate racial patterns for uterine cancer in relation to histologic subtype and stage at diagnosis. In this context, we have compared the incidence and mortality trends in the state of Michigan for black and white women with cancers of the corpus uteri diagnosed during the years 1985-1994.

\section{METHODS}

\section{Study Population and Eligibility}

The study population was obtained from the Michigan cancer incidence and all-cause mortality registries, collected by the Cancer Surveillance Program of the Michigan Department of Community Health. Eligibility for inclusion in the cancer incidence registry was limited to newly diagnosed invasive cancers with the "corpus uteri" topography described in the 1976 International Classification of Diseases for Oncology (ICD-0). ${ }^{13}$ From the mortality registry, eligibility was limited to deaths of female residents of the state of Michigan, where cause was coded as "malignant neoplasm of the corpus uteri" (codes 182.0-182.8) or "uterus, not otherwise specified" (code 179.0) using the International Classification of Diseases, 9th revision. ${ }^{14}$ Since the 1980 s, at least $75 \%$ of deaths from cancer with a topography coded as "uterus, not otherwise specified" have been subsequently confirmed on medical record review to have been caused by cancer of the uterine corpus. ${ }^{15}$ Overall, the study population included 11,146 white, 879 black, and 54 women of other races from the cancer incidence registry and 1986 white, 324 black, and 15 women of other races from the mortality registry. To assess the racial associations of interest, both incidence and mortality data were selected to include only black or white cases.

\section{Variables}

Covariates from the incidence registry were race, year of diagnosis, histologic subtype, age group, and stage at diagnosis. Stage was classified as localized (confined to the organ of origin), regional (direct extension to adjacent organs or tissues or lymph node involvement), distant (spread to nonadjacent organs, distant metastasis, or involvement of distant lymph nodes), or unstaged. ${ }^{16}$ In addition, stage was collapsed into two categories for fitting as a dichotomous outcome: advanced stage (regional or distant) and not advanced stage (localized). A derived variable was constructed for age, collapsing age into two groups: younger than 65 years and age 65 or older. The mean ages were calculated using the midpoint of each 5-year age group as each patient's age. Histologic subtype was classified using ICD-0, and derived variables were created that collapsed subtypes into the following categories: ${ }^{17}$ 1) adenocarcinoma, 2) adenoacanthoma, 3) adenosquamous carcinoma, 4) clear cell adenocarcinoma, 5) serous adenocarcinoma, 6) mucinous adenocarcinoma, 7) epithelial (undifferentiated) carcinoma, 8) squamous cell carcinoma, 9) all sarcomas, and 10) other histology. In addition, based on previous classifications of the aggressiveness of various histologic subtypes, ${ }^{12,18-20}$ categories were created for less aggressive adenocarcinomas (adenocarcinoma, mucinous adenocarcinoma, adenoacanthoma, and adenosquamous adenocarcinoma), more aggressive adenocarcinomas (clear cell adenocarcinoma, serous adenocarcinoma, squamous carcinoma, and undifferentiated carcinoma), sarcomas, and other histology. A second category for more aggressive adenocarcinomas was also created, with the subtype squamous carcinoma dropped. Our concern was that, because squamous carcinoma rarely occurs in its pure form, ${ }^{20}$ the squamous category might be subject to a high level of misclassification due to either incomplete tumor sampling or the presence of a cervical primary.

From the mortality registry, the variables age at 
death and race were included. As with incidence data, age was divided into two groups: younger than 65 years and age 65 years or older.

\section{Analysis/Statistical Methods}

Cumulative incidence and mortality rates were calculated for 1985-1989 and 1990-1994, and for the 10year period overall. For all rates, the denominator used was the Michigan female population at the midpoint of each time period (i.e., 1987 for the 1985-1989 rates, 1992 for the 1990-1994 rates, and 1990 for the overall rates $\left.{ }^{21-23}\right)$. All rates were age-adjusted, using the direct method, to the 1970 U.S. population standard. ${ }^{24}$ Because the state registry data accounted for $95 \%$ of all incident cancer cases and virtually all cancer deaths that occurred in the Michigan population between 1985 and 1994, confidence intervals (CIs) for rates and ratios were not required.

All analyses were performed using the SAS (Statistical Analysis Software) package (SAS Institute Inc., Cary, NC). Associations among race, age, histologic subtype, and stage were first assessed by univariate analysis using the Mantel-Haenszel chi-square test. Stratified analyses were then performed using the Mantel-Haenszel method, and the presence of interaction was assessed using the Breslow-Day test. Variables associated with stage in the univariate analysis were subsequently included in a logistic regression model to determine which factors were independent predictors of stage. The significance of each model was assessed with a likelihood ratio test. For odds ratios obtained from logistic regression, 95\% confidence intervals were calculated. For Cochran-MantelHaenszel odds ratios, corresponding 95\% test-based CIs were obtained. Differences between the mean ages for each histologic subtype, overall and according to race, were evaluated using nonparametric tests. Specifically, we used Wilcoxon's rank sum test to analyze ranks of data containing two levels and the KruskalWallis test to analyze ranks when more than two levels were present. $^{25}$

\section{RESULTS}

\section{Incidence and Mortality Rates}

The overall 10-year age-adjusted incidence rate for cancer of the corpus uteri per 100,000 women was 21.99 , and the overall age-adjusted mortality rate per 100,000 women was 3.82 . The trends in incidence and mortality rates are summarized in Table 1. Although the overall age-adjusted incidence rate was relatively stable between the two 5-year periods, the age-adjusted incidence rate per 100,000 increased for women age 65 years or older from 97.67 in 1985-1989 to 103.07 in 1990-1994, due primarily to an increasing inci- dence for older white women. Incidence rates remained relatively stable for black women. In comparison to white women, black women had a $40 \%$ lower risk (rate ratio $[R R]=0.60$ ) of developing cancer of the corpus uteri.

Mortality rates per 100,000 improved for the most recent 5 years, where rates decreased for both age groups as well as for both race groups. For white women, the decrease held for both age groups, whereas for black women, only the older age group experienced a decrease. In Michigan, black women had $54 \%$ greater risk $(\mathrm{RR}=1.54)$ of dying, and this excess risk increased during the most recent 5 years (from $51 \%$ to $63 \%$ ) due to an increase for younger black women.

The age specific incidence rates by race for Michigan compared with the U.S. SEER rates ${ }^{1}$ are presented in Figure 1. The age specific incidence patterns in Michigan during the years 1985-1994 tended to parallel those observed in the U.S. during 1988-1992, and the age-adjusted incidence rate per 100,000 was 21.99 in Michigan compared with 21.3 for the entire U.S. Mortality rates in Michigan were also similar to those in the U.S.; the overall age-adjusted mortality rate per 100,000 in Michigan during the 10-year study period was 3.8 , compared with 3.4 in the U.S. during the years 1988-1992. ${ }^{1}$ Age specific mortality rates for Michigan during the years 1985-1994 were compared with U.S. SEER age-specific mortality rates for 1988-1992 (Fig. 2). For each age specific group, the mortality rate in Michigan was higher than that in the U.S. for white women, especially for the older age groups. For black women, the mortality rate in Michigan was lower than that in the U.S., with the lower rates most pronounced in the older age groups.

\section{Variables Associated with Prognosis}

The distribution by age (age $<65$ years or $\geq 65$ years) did not differ between black and white women (Table 2). The distribution by stage at diagnosis in Michigan was consistent with trends in the U.S.; black women, compared with white women, were diagnosed with more advanced stage disease. During the 10-year period, only $49.4 \%$ of black women were diagnosed with localized disease, compared with $68.5 \%$ of white women; and $17.6 \%$ of black women manifested distant disease at diagnosis, compared with only $6.5 \%$ of white women.

During the 10-year study period, the predominant histologic subtypes for all races were adenocarcinomas. Black women had relatively fewer adenocarcinomas than white women (62.6\% vs. $80.4 \%)$. In addition, black women had fewer of the histologic variants of adenocarcinoma known to be associated with a favor- 
TABLE 1

Age-Adjusted Incidence and Mortality Rates for Cancer of the Corpus Uteri, by Race, for Michigan Residents, $1985-1994^{\text {a }}$

\begin{tabular}{|c|c|c|c|c|}
\hline & All races & White & Black & $\begin{array}{l}\text { Rate ratio } \\
\text { (Black:White) }\end{array}$ \\
\hline \multicolumn{5}{|l|}{ Incidence } \\
\hline \multicolumn{5}{|c|}{ All yrs (1985-1994) } \\
\hline All ages & 21.99 & 23.22 & 13.95 & 0.60 \\
\hline Age $<65$ yrs & 13.48 & 14.44 & 7.68 & 0.53 \\
\hline Age $\geq 65$ yrs & 99.61 & 103.34 & 71.12 & 0.69 \\
\hline \multicolumn{5}{|l|}{$1985-1989$} \\
\hline No. $(\%)$ & 5754 & $5322(92.5 \%)$ & $412(7.2 \%)$ & \\
\hline All ages & 21.51 & 22.50 & 14.12 & 0.63 \\
\hline Age $<65$ yrs & 13.16 & 13.93 & 7.68 & 0.55 \\
\hline Age $\geq 65$ yrs & 97.67 & 100.76 & 72.89 & 0.72 \\
\hline \multicolumn{5}{|l|}{ 1990-1994 } \\
\hline No. $(\%)$ & 6325 & $5824(92.1 \%)$ & 467 (7.4\%) & \\
\hline All ages & 22.35 & 23.58 & 14.17 & 0.60 \\
\hline Age $<65$ yrs & 13.51 & 14.44 & 7.71 & 0.53 \\
\hline Age $\geq 65$ yrs & 103.07 & 107.01 & 73.15 & 0.68 \\
\hline \multicolumn{5}{|l|}{ Mortality } \\
\hline \multicolumn{5}{|c|}{ All yrs (1985-1994) } \\
\hline All ages & 3.82 & 3.61 & 5.55 & 1.54 \\
\hline Age $<65$ yrs & 1.43 & 1.34 & 2.08 & 1.55 \\
\hline Age $\geq 65$ yrs & 25.67 & 24.38 & 37.27 & 1.53 \\
\hline \multicolumn{5}{|l|}{ 1985-1989 } \\
\hline No. $(\%)$ & 1180 & 1009 (85.5\%) & $167(14.2 \%)$ & \\
\hline All ages & 3.98 & 3.79 & 5.74 & 1.51 \\
\hline Age $<65$ yrs & 1.53 & 1.46 & 2.07 & 1.42 \\
\hline Age $\geq 65$ yrs & 26.34 & 25.06 & 39.18 & 1.56 \\
\hline \multicolumn{5}{|l|}{ 1990-1994 } \\
\hline No. $(\%)$ & 1175 & 977 (83.1\%) & 187 (15.9\%) & \\
\hline All ages & 3.66 & 3.41 & 5.57 & 1.63 \\
\hline Age $<65$ yrs & 1.30 & 1.17 & 2.10 & 1.79 \\
\hline Age $\geq 65$ yrs & 25.19 & 23.78 & 37.23 & 1.57 \\
\hline
\end{tabular}

able prognosis, including adenoacanthoma $(0.7 \%$ vs. $3.0 \%)$ and mucinous adenocarcinoma ( $0.2 \%$ vs. $1.0 \%)$. Black women had a greater proportion of adenocarcinoma subtypes known to be associated with an unfavorable prognosis, including clear cell (3.7\% vs. $1.3 \%)$ and serous (3.3\% vs. $1.2 \%)$. Black women also had a higher proportion of sarcomas than white women ( $18.0 \%$ vs. $5.6 \%)$.

The mean age at diagnosis was significantly different across each of the four broad histologic subtypes (Kruskal-Wallis chi-square $=105.61$, degrees of freedom $[\mathrm{df}]=3, P=0.0001)$. Moreover, women with more aggressive histologic subtypes were older than women with less aggressive histologic subtypes (Wilcoxon two-sample normal approximation z-statistic standard score $=9.67, P=0.0001$ ). However, the mean age at diagnosis by histologic subtype between races did not differ, and an evaluation of the mean age at diagnosis for each particular histologic subtype demonstrated no differences between black and white women for any of the subtypes (Wilcoxon two-sample rank sum tests, $P$ values ranged from 0.12 to 0.69 ).

Overall, more aggressive histologic subtypes were associated with more advanced stages. Most notable, however, was the pattern for black women. With the exception of mucinous adenocarcinoma, the trend was for black women to have more advanced stage disease within each histologic subtype. However, because of the small numbers of black women in several of these subgroups, only three of these trends reached statistical significance (adenocarcinoma, $P=0.001$; adenosquamous carcinoma, $P<0.05$; sarcoma, $P<$ 0.05).

Mantel-Haenszel chi-square tests conducted to evaluate associations between race and the prognostic factors confirmed the trends presented in Table 2. Age at diagnosis did not differ between black and white women. However, compared with white women, black 

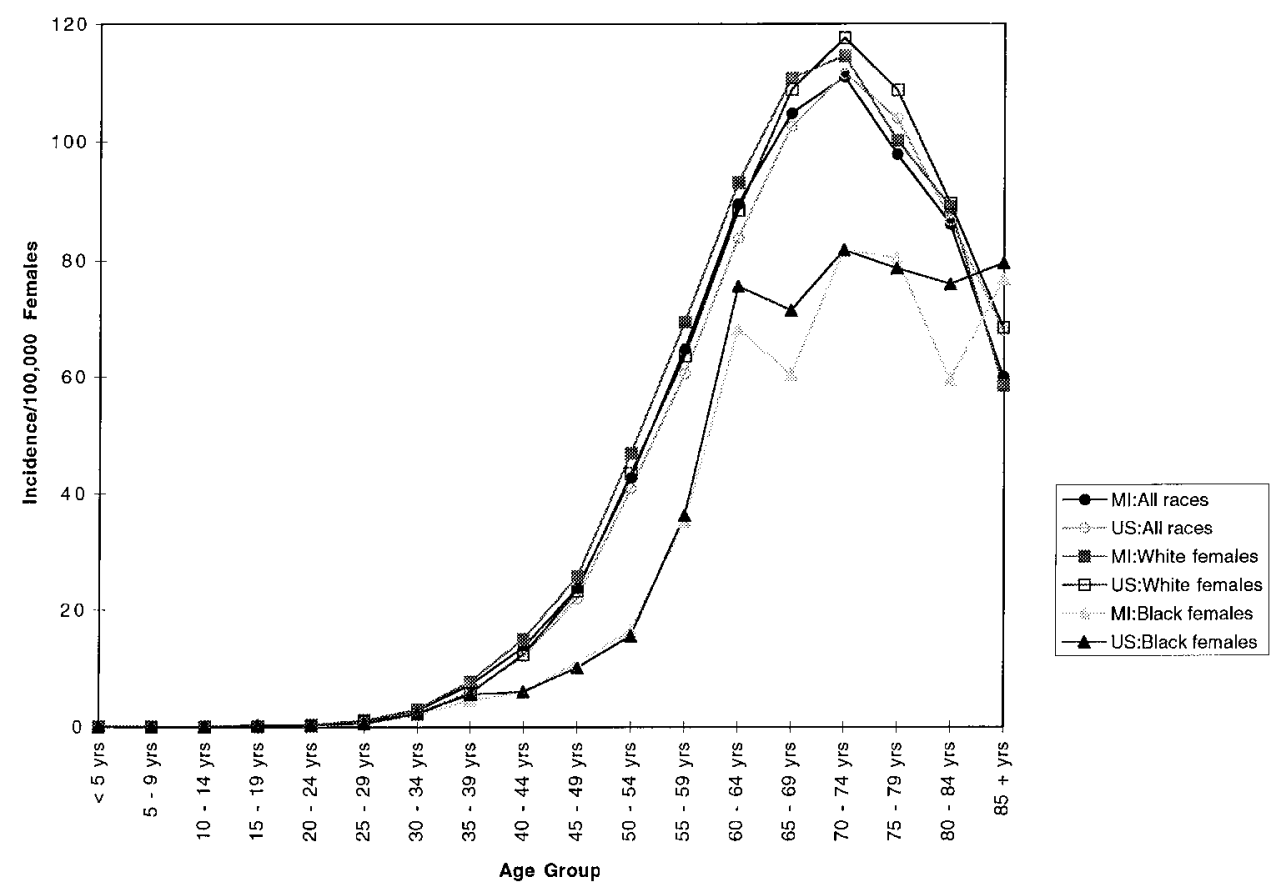

FIGURE 1. Age specific incidence, rates by race, uterine corpus cancer, U.S. SEER, 1988-92, and Michigan, 1985-94.

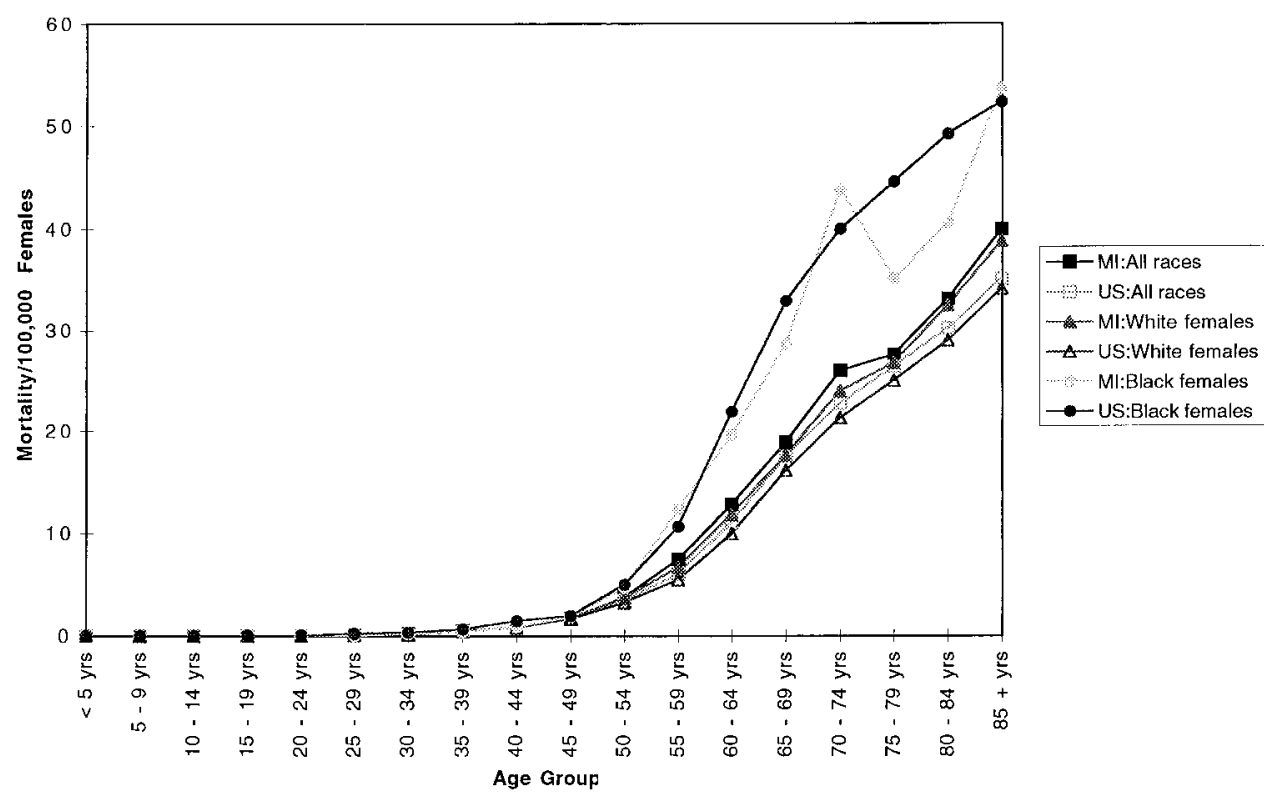

FIGURE 2. Age specific mortality, rates by race, uterine corpus cancer, U.S. SEER, 1988-92, and Michigan, 1985-94.

women were two and one-half times more likely to present with advanced stage disease, two and one-half times more likely to present with more aggressive adenocarcinomas, and three and one-half times more likely to present with sarcomas (Table 3). When the analysis was repeated with the subtype of squamous carcinoma dropped from the category of more aggressive adenocarcinomas, the results were virtually identical. Black women were still two and one-half times more likely to present with more aggressive adenocarcinomas (odds ratio $[\mathrm{OR}]=2.55,95 \% \mathrm{CI}=2.08-3.12$ ).
Age-adjusted incidence rates per 100,000 were calculated to compare absolute numbers of histologic subtypes occurring in black and white women. The incidence rate per 100,000 of less aggressive adenocarcinomas was 20.52 for white women and 9.39 for black women (black:white $[\mathrm{B}: \mathrm{W}] \mathrm{RR}=0.46$ ). For more aggressive adenocarcinomas, the rate per 100,000 was 1.29 for white women and 1.84 for black women (B:W $\mathrm{RR}=1.43$ ); for sarcomas, the rate per 100,000 was 1.28 for white women and 2.48 for black women (B:W RR = 1.94); and for other subtypes, the rate per 100,000 was 
TABLE 2

Summary of Age and Tumor Characteristics for Cancer of the Corpus Uteri, by Race, for Michigan Residents, 1985-1994

\begin{tabular}{lllll}
\hline & $\begin{array}{l}\text { Overall } \\
\text { No. (\%) }\end{array}$ & $\begin{array}{l}\text { White } \\
\text { No. (\%) }\end{array}$ & $\begin{array}{l}\text { Black } \\
\text { No. (\%) }\end{array}$ & $\begin{array}{l}\text { Other } \\
\text { No. (\%) }\end{array}$ \\
\hline $\begin{array}{lllll}\text { Age at diagnosis } \\
\quad \text { 65 yrs }\end{array}$ & $5661(46.9)$ & $5220(46.8)$ & $407(46.3)$ & $34(63.0)$ \\
$\begin{array}{l}\geq 65 \text { yrs } \\
\text { Stage at diagnosis }\end{array}$ & $6418(53.1)$ & $5926(53.2)$ & $472(53.7)$ & $20(37.0)$ \\
$\quad$ Localized & $8103(67.1)$ & $7635(68.5)$ & $434(49.4)$ & $34(63.0)$ \\
Regional & $1689(14.0)$ & $1478(13.3)$ & $198(22.5)$ & $13(24.1)$ \\
Distant & $886(7.3)$ & $728(6.5)$ & $155(17.6)$ & $3(5.5)$ \\
Unstaged & $1401(11.6)$ & $1305(11.7)$ & $92(10.5)$ & $4(7.4)$ \\
Histologic subtype & & & & \\
Adenocarcinoma & $9559(79.1)$ & $8970(80.4)$ & $550(62.6)$ & $39(72.2)$ \\
$\quad$ Adenoacanthoma & $337(2.8)$ & $330(3.0)$ & $6(0.7)$ & $1(1.9)$ \\
$\quad$ Adenosquamous & $411(3.4)$ & $380(3.4)$ & $29(3.3)$ & $2(3.7)$ \\
$\quad$ Clear cell & $184(1.6)$ & $150(1.3)$ & $33(3.7)$ & $1(1.8)$ \\
$\quad$ Serous & $165(1.4)$ & $134(1.2)$ & $29(3.3)$ & $2(3.7)$ \\
$\quad$ Mucinous & $110(0.9)$ & $107(1.0)$ & $2(0.2)$ & $1(1.9)$ \\
$\quad$ Undifferentiated & & & & \\
$\quad$ carcinoma & $320(2.6)$ & $286(2.6)$ & $34(3.9)$ & $0(0.0)$ \\
Squamous carcinoma & $121(1.0)$ & $98(0.9)$ & $23(2.6)$ & $0(0.0)$ \\
Sarcomas (all) & $794(6.6)$ & $629(5.6)$ & $158(18.0)$ & $7(13.0)$ \\
Other & $78(0.6)$ & $62(0.6)$ & $15(1.7)$ & $1(1.8)$ \\
\hline
\end{tabular}

0.15 for white women and 0.24 for black women (B:W $\mathrm{RR}=1.60$ ).

To compare absolute numbers of stage at diagnosis (advanced vs. localized) for black and white women, age-adjusted incidence rates per 100,000 were calculated. For localized stage disease, the rate per 100,000 was 16.16 for white women and 6.90 for black women ( $\mathrm{B}: \mathrm{W} R \mathrm{RR}=0.43)$. For advanced stage disease, the rate per 100,000 was 4.46 for white women and 5.60 for black women (B:W RR $=1.26$ ).

Age at diagnosis and histologic subtype were both associated with stage at diagnosis. Older women were more likely to present with advanced stage disease (chi-square $=80.64, \mathrm{df}=1, P=0.001$ ). Women with more aggressive adenocarcinomas, sarcomas, and histologic subtypes classified as "other" were more likely to present with advanced stage disease (chi-square = 348.98, df $=1, P=0.001$ ) than women with less aggressive adenocarcinomas. A significant interaction between race and a combined variable for all histologic subtypes representing more aggressive tumors was found that modified the association between histologic subtype and advanced stage (Breslow-Day chisquare $=5.23$, df $=1, P=0.02$ ). After a separate assessment of each subtype for any interaction with race, a borderline significant interaction was found between more aggressive adenocarcinomas and race (Breslow-Day chi-square $=3.375$, df $=1, P=0.07$ ). Specifically, the risk of advanced stage disease, given a histologic subtype of more aggressive adenocarcinomas compared with less aggressive adenocarcinomas, was greater for white women $(\mathrm{OR}=3.42, P<0.001)$ than for black women $(\mathrm{OR}=2.22, P<0.001)$. Conversely, advanced stages were exhibited more frequently for black women with less aggressive adenocarcinomas.

The logistic regression modeling strategy involved several steps (Table 4). The crude association was first estimated, then other prognostic factors found to be associated with advanced stage in univariate analyses were added to evaluate whether the crude association changed. When both age and histologic subtype were added simultaneously, the risk of advanced stage for black women decreased ( $\mathrm{OR}=2.37, P<0.0001)$; and based upon the likelihood ratio (LR) test, this was the best model. Although the model that included race, age, histologic subtype, and the interaction between race and histologic subtype was not statistically superior to the model without the interaction term based on the likelihood ratio test $(\mathrm{LR}=4.52, \mathrm{df}=3, P>$ 0.10 ), the magnitude of the OR changed significantly when the interaction term was included. Therefore, the final accepted model was the one that adjusted for age, histologic subtype, and the interaction between race and histologic subtype, where black women had a risk of presenting with advanced stage disease that was over two and one-half times the risk for white women $(\mathrm{OR}=2.63,95 \% \mathrm{CI}=2.19-3.16)$. Results were identical when the subtype squamous carcinoma was dropped from the "more aggressive adenocarcinomas" category and the final model was run again $(\mathrm{OR}=2.67)$.

\section{DISCUSSION}

U.S. SEER data has demonstrated that cancer of the corpus uteri occurs more commonly among white women, yet 5-year relative survival within each stage of disease is poorer and age-adjusted mortality is higher for black women. ${ }^{1}$ Several clinic-based ${ }^{2,5}$ and population-based ${ }^{4,7}$ studies of incident cases from the 1970s and 1980s have documented a lower survival rate for black women with cancer of the corpus uteri. To explore whether black-white patterns for incidence and mortality from cancer of the corpus uteri were changing, we compared incidence and mortality in the 1990s with incidence and mortality in the 1980s. In the 1990s, incidence for white women, especially those age 65 years or older, increased by $6.2 \%$. Overall mortality rates decreased slightly for both races, although mortality rates for black women younger than 65 years did not change. This represents a reversal of the trends from the 1970s and 1980s. Specifically, U.S. SEER data collected between 1973 and 1987 for cancer of the 
TABLE 3

Summary of Univariate Analyses of Age and Tumor Characteristics for Cancer of the Corpus Uteri, by Race, for Michigan Residents, 1985-1994

\begin{tabular}{|c|c|c|c|}
\hline Characteristic & $\begin{array}{l}\% \text { White } \\
(\mathrm{n}=11,146)\end{array}$ & $\begin{array}{l}\% \text { Black } \\
(\mathrm{n}=879)\end{array}$ & $\begin{array}{l}\text { Odds ratio }(95 \% \\
\text { confidence interval) } \\
\text { (black vs. white) }\end{array}$ \\
\hline \multicolumn{4}{|l|}{ Age at diagnosis } \\
\hline$<65$ yrs & 46.9 & 46.3 & Reference \\
\hline$\geq 65 \mathrm{yrs}$ & 53.2 & 53.7 & $1.02(0.90-1.16)$ \\
\hline \multicolumn{4}{|l|}{ Stage at diagnosis } \\
\hline Not advanced & 68.5 & 49.4 & Reference \\
\hline Advanced & 19.8 & 40.1 & $2.57(2.25-2.92)$ \\
\hline No. of patients ${ }^{\mathrm{a}}$ & 9841 & 787 & \\
\hline \multicolumn{4}{|l|}{ Histologic subtype } \\
\hline Less aggressive adenocarcinomas & 87.8 & 66.8 & Reference \\
\hline More aggressive adenocarcinomas & 6.0 & 13.5 & $2.67(2.23-3.21)$ \\
\hline Sarcomas & 5.6 & 18.0 & $3.55(3.03-4.16)$ \\
\hline Other & 0.6 & 1.7 & $3.44(2.16-5.49)$ \\
\hline
\end{tabular}

TABLE 4

Summary of Logistic Regression Modeling of Significant Predictors of Advanced Stage Disease for Cancer of the Corpus Uteri in Black Women, Michigan Residents, 1985-1994

\begin{tabular}{|c|c|c|c|}
\hline Model & $\begin{array}{l}\text { Likelihood ratio } \\
\text { statistic (df) }\end{array}$ & $\begin{array}{l}\text { Odds ratio for } \\
\text { advanced } \\
\text { stage (black } \\
\text { vs. white); }\end{array}$ & $P$ value \\
\hline 1. Race alone & $11,556.009(\mathrm{df}=1)$ & 2.82 & $<0.0001$ \\
\hline 2. Race and age ( $\geq 65 \mathrm{yrs})$ & $11,474.537(\mathrm{df}=2)$ & 2.83 & $<0.0001$ \\
\hline 3. Race and histology & $11,297.79(\mathrm{df}=4)$ & 2.36 & $<0.0001$ \\
\hline 4. Race, age, and histology & $11,225.643(\mathrm{df}=5)$ & 2.37 & $<0.0001$ \\
\hline $\begin{array}{l}\text { 5. Race, age, histology, and } \\
\text { interaction between race }\end{array}$ & 11.221 .12 & 263 & $<0.0001$ \\
\hline
\end{tabular}

corpus uteri demonstrated stable incidence for black women but a decrease in incidence for white women from 30.8 to 23.0 per $100,000 .{ }^{26}$ Moreover, from the 1970 s to the 1980 s, U.S. SEER data demonstrated consistent decreases in age-specific mortality rates from cancer of the corpus uteri for black and white women. ${ }^{1}$

The recent shift in incidence and mortality trends, in which incidence has increased proportionately more for white women but mortality has decreased proportionately less for black women, may be due to differences in the distribution of several factors that may affect risk, including socioeconomic status and cultural factors associated with access to and quality of preventive practices and medical care, as well as life-style risk factors. ${ }^{27}$ The increase in the incidence rate from 100.76 during the years 1985-1989 to 107.01 during the years 1990-1994 for white women age 65 years or older was probably not due solely to increased exposure to unopposed estrogen-replacement therapy (ERT) ${ }^{28}$ A conclusion of the Postmenopausal Estrogen/Progestin Interventions (PEPI) Trial was that physicians should give serious consideration to the addition of progestin when administering ERT to postmenopausal women with an intact uterus; ${ }^{29}$ in addition, it has been noted that progestins are now generally added to estrogen regimens given to women (without prior hysterectomy) to reduce the risk of endometrial cancer associated with unopposed estrogen therapy. ${ }^{28}$ However, the increase in incidence warrants increased clinical surveillance of older high risk white women to identify uterine cancers at the earliest stage possible.

Like the authors of other reports, ${ }^{28,30-32}$ we observed an association between older age at diagnosis and the incidence and percentage frequency of more aggressive histologic subtypes, including clear cell adenocarcinoma, serous adenocarcinoma, and undifferentiated carcinoma. The increase in more aggressive histologic subtypes occurring in older women may be accompanied by the accumulation of genetic mutations that are expressed in estrogen-independent tumor progression. For example, p53 abnormalities have been demonstrated in $86 \%$ of serous adenocarcinomas, compared with only $20 \%$ of endometrioid adenocarcinomas. ${ }^{10}$ For other aggressive histologic subtypes, consistent molecular genetic and epigenetic alterations have yet to be identified. There were no differences between blacks and whites in mean age at diagnosis, either within each histologic subtype or across subtypes. 
Our results were consistent with data for the U.S., ${ }^{1}$ in that stage was more advanced for black women. Black women also had higher proportions of more aggressive histologic subtypes, including clear cell adenocarcinomas, serous adenocarcinomas, and sarcomas, and their risk of presenting with more aggressive histologic subtypes was between two and one-half to three and one-half times greater. Like the authors of a recent, similar study, ${ }^{33}$ we observed that the incidence rate per 100,000 of the relatively infrequent but more aggressive subtypes of endometrial carcinoma was similar between black (1.84) and white (1.29) women, and that the $40 \%$ decrease in age-adjusted incidence among black women was due to a lower absolute risk of 11 cases per 100,000 for less aggressive histologic subtypes.

In our multivariate model, black women still had a risk of advanced stage disease two and one-half times greater than that of white women. A notable finding was that black women were more likely to have more advanced stage disease within each histologic subtype; this brings into question the inference that the increased frequency of advanced stage disease in black women is primarily a consequence of experiencing more aggressive histologic subtypes. Moreover, we observed a difference in the incidence rate per 100,000 of advanced stage disease at diagnosis between black (5.60) and white (4.46) women, likewise suggesting a higher absolute risk of advanced stage disease for black women.

Because of our concern that sarcomas may have a different association with to stage at diagnosis than endometrial carcinomas, we repeated the multivariate analysis and excluded women with sarcomas. The results were completely identical in terms of our analytic predictive Model 5 (OR 2.63, $P<0.0001$, LR statistic 10293.180, $\mathrm{df}=6$ ), and the only noteworthy difference was for Model 4 (race, age, and histology), where the risk of advanced stage for black women compared with white women increased from 2.37 to 2.47 when sarcomas were excluded.

Although this study was able to assess several prognostic factors for cancer of the corpus uteri and the differences in these factors between black and white women, there were some limitations. In calculating incidence rates, the denominator should only include the population at risk. Because the rate of hysterectomy has been shown to be as high as $35 \%$ for women age 60 or older, ${ }^{34}$ including all of the female population in Michigan in the denominator resulted in underestimation of the incidence rates. However, SEER incidence rates were also based on the entire female population in specific regions of the U.S.

The structure and content of the registry data also imposed limitations. One significant limitation was the inability to link cancer incidence with all-cause mortality, making it impossible to evaluate the prognostic effect of stage at diagnosis or histologic subtype on racial differences in mortality. In addition, tumor grade was not included in the registry data, eliminating our ability to include this important prognostic variable in our analyses. However, we likely would have found tumor grade to be highly correlated with our category of more aggressive adenocarcinomas. This category was comprised of clear cell, serous, and undifferentiated carcinomas; these are typically high grade lesions ${ }^{19,33,35}$ for which grading is based on nuclear grade rather than architectural grade, and they are, in fact, frequently ungraded. ${ }^{31,36}$

This study also had several strengths. The sample size was large, allowing for adequate stratification of the data without compromising the total numbers of cases in each subdivision. In addition, because the data were obtained from a large population-based registry, which contained information on all incident cases of cancer of the corpus uteri and all deaths attributed to cancer of the corpus uteri in the state of Michigan, it should be possible to apply the results generally to other U.S. states that have similar sociodemographic distributions.

We were able to conduct a comprehensive evaluation of black-white differences by histologic subtype in the context of evaluating differences in stage between black and white women. We demonstrated that, although black women experienced a greater frequency of adverse histopathologic features, including sarcomas, these more aggressive forms of cancer of the corpus uteri accounted for only $10 \%$ of the excess risk for advanced stage disease at diagnosis for black women (using the formula $\left\{R_{\text {unadjusted }}-R_{\text {adjusted }}\right\}$ / $\left\{R_{\text {unadjusted }}-1.0\right\}^{37}$ ). In fact, the interaction term demonstrated that histologic subtype was more predictive of advanced stage disease for white women than it was for black women. This suggests that there are other important determinants of stage for black women. The intuitive hypothesis for explaining these differences is that black women may have less access to care on symptom recognition or may delay seeking treatment after the onset of symptoms. However, studies conducted to evaluate delay in seeking treatment have not found significant differences between black and white women. ${ }^{3,8,9}$ Perhaps other correlates or predictors of advanced stage or of mortality among black women should be explored, such as the molecular determinants of multistep progression of the cancer; life-style and psychosocial risk factors, including comorbidity at the time of diagnosis; and barriers to access and utilization of optimal health care. 


\section{REFERENCES}

1. Ries LAG, Kosary CL, Hankey BF, Miller BA, Harras A, Edwards BK, editors. SEER Cancer Statistics Review, 19731994: tables and graphs. NIH Pub. No. 96-2789. Bethesda, MD: National Cancer Institute, 1997.

2. Aziz H, Rotman M, Hussain F, Smith G, Chan E, Choi K, et al. Poor survival of black patients in carcinoma of the endometrium. Am J Radiat Oncol Biol Phys 1993;27:293-301.

3. Barrett RJ, Harlan LC, Wesley MN, Hill HA, Chen VW, Clayton LA, et al. Endometrial cancer: stage at diagnosis and associated factors in black and white patients. Am J Obstet Gynecol 1995;173:414-23.

4. Bain RP, Greenberg RS, Chung KC. Racial differences in survival of women with endometrial cancer. Am J Obstet Gynecol 1987;157:914-23.

5. Cronje HS, Fourie S, Doman MJ, Helms JB, Nel JT, Goedhals L. Racial differences in patients with adenocarcinoma of the endometrium. Int J Gynaecol Obstet 1992;39:213-8.

6. Hill HA, Coates RJ, Austin H, Correa P, Robboy SJ, Chen V, et al. Racial differences in tumor grade among women with endometrial cancer. Gynecol Oncol 1995;56:154-63.

7. Hill HA, Eley JW, Harlan LC, Greenberg RS, Barrett RJ II, Chen VW. Racial differences in endometrial cancer survival: the black/white cancer survival study. Obstet Gynecol 1996; 88:919-26.

8. Liu JR, Conaway M, Rodriguez GC, Soper JT, Clarke-Pearson DL, Berchuck A. Relationship between race and interval to treatment in endometrial cancer. Obstet Gynecol 1995;86: 486-90.

9. Coates RJ, Click LA, Harlan LC, Robboy S, Barrett RJ, Eley JW, et al. Differences between black and white patients with cancer of the uterine corpus in interval from symptom recognition to initial medical consultation (United States). Cancer Causes Control 1996;7:328-36.

10. Sherman ME, Sturgeon S, Brinton L, Kurman RJ. Endometrial cancer chemoprevention: implications of diverse pathways of carcinogenesis. J Cell Biochem Suppl 1995;23:160-4.

11. Nyholm HCJ, Nielsen AL, Norup P. Endometrial cancer in postmenopausal women with and without previous estrogen replacement therapy: comparison of clinical and histopathological characteristics. Gynecol Oncol 1993;49:22935.

12. Rose, PG. Endometrial carcinoma. N Engl J Med 1996;335: $640-9$.

13. Percy C, VanHolten V, Muis C, editors. The international classification of diseases for oncology. Geneva: World Health Organization, 1976.

14. International Classification of diseases, ICD-9-CM (9th revision, clinical modification), 4th edition. Los Angeles: Practice Management Information Corporation, 1995.

15. Grady D, Ernster VL. Endometrial cancer. In: Schottenfeld D, Fraumeni JF Jr. Cancer epidemiology and prevention. New York: Oxford University Press, 1996: 1058-89.

16. Summary staging guide, SEER Program, January 1981. NIH Publication No. 81. Bethesda, MD: National Cancer Institute, 1981:1313.

17. Beahrs OH, Henson DE, Hutter RVP, Kennedy BJ, editors. Manual for staging of cancer. 4th edition. Philadelphia: J. B. Lippincott, 1992.
18. Hoskins WJ, Perez CA, Young RC. Principles and practice of gynecologic oncology. Philadelphia: J. B. Lippincott, 1992.

19. Scully RE, Bonfiglio TA, Kurman, RJ, et al., editors. Histologic typing of female genital tract tumors. In: World Health Organization. International histologic classification of tumours, 2nd edition. New York: Springer-Verlag, 1994.

20. Shingleton HM, Fowler WC, Jordan JA, Lawrence WD. Gynecologic oncology: current diagnosis and treatment. London: W. B. Saunders, 1996.

21. Michigan Department of Public Health. Michigan health statistics, 1987. Lansing, MI: Office of State Registrar, Division of Health Statistics, 1989:324.

22. Michigan Department of Public Health. Michigan health statistics, 1992. Lansing, MI: Office of State Registrar, Division of Health Statistics, 1993:235.

23. Michigan Department of Public Health. Michigan health statistics, 1994. Lansing, MI: Office of State Registrar, Division of Health Statistics 1995:229.

24. Kurian GT. Datapedia of the U.S., 1790-2000, America year by year. Lanham, MD: Bernan Press, 1994.

25. Cody RP, Smith JK. Applied statistics and the SAS programming language. 2nd edition. New York: Elsevier, 1987.

26. Platz CE, Benda JA. Female genital tract cancer. Cancer 1995;75(Suppl 1):270-94.

27. Schottenfeld D. Determinants of black/white survival differences among women with endometrial cancer [editorial]. Gynecol Oncol 1995;56:151-3.

28. Grady D, Gebretsadik T, Kerlikowske K, Ernster V, Petitti D. Hormone replacement therapy and endometrial cancer risk: a meta-analysis. Obstet Gynecol 1995;85:304-13.

29. The Writing Group for the PEPI Trial. Effects of hormone replacement therapy on endometrial histology in postmenopausal women: the postmenopausal estrogen/progestin interventions (PEPI) trial. JAMA 1996;275:370-5.

30. Burke TW, Fowler WC, Morrow CP. Clinical aspects of risk in women with endometrial carcinoma. J Cell Biochem Suppl 1995;23:31-6.

31. Abeler VM, Vergote IB, Kjorstad KE, Trope CG. Clear cell carcinoma of the endometrium: prognostic and metastatic pattern. Cancer 1996;78:1740-7.

32. Schottenfeld D. Epidemiology of endometrial neoplasia. J Cell Biochem Suppl 1995;23:151-9.

33. Plaxe SC, Saltzstein, SL. Impact of ethnicity on the incidence of high-risk endometrial carcinoma. Gynecol Oncol 1997;65: $8-12$.

34. Howe HL. Age-specific hysterectomy and oophorectomy prevalence rates and the risks for cancer of the reproductive system. Am J Public Health 1984;74:560.

35. Wilson TO, Podratz KC, Gaffey TA, Malkasian GD, O’Brien PC, Naessens JM. Evaluation of unfavorable histologic subtypes in endometrial adenocarcinoma. Am J Obstet Gynecol 1990;162:418-26.

36. Matthews RP, Hutchinson-Colas J, Maiman M, Fruchter RG, Gates EJ, Gibbon D, et al. Papillary serous and clear cell type lead to poor prognosis of endometrial carcinoma in black women. Gynecol Oncol 1997;65:206-12.

37. Otten MW, Teutsch SM, Williamson DF, Marks JS. The effect of known risk factors on excess mortality of black adults in the United States. JAMA 1990;263:845-50. 